\title{
Active Longitudinal Structures of the Sun from MDI and EIT Observations
}

\author{
E. E. Benevolenskaya
}
Pulkovo Astronomical Observatory, St. Petersburg, Russia
W.W.Hansen Experimental Physics Laboratory, Stanford University, Stanford, CA, USA

\begin{abstract}
A. G. Kosovichev and P. H. Scherrer
W.W.Hansen Experimental Physics Laboratory, Stanford University, Stanford, CA, USA
\end{abstract}

\begin{abstract}
We present results of investigation of the large-scale structure of the solar corona during the transition period between solar cycles 22 and 23 and at the beginning of the current cycle 23 using the SOHO/EIT EUV data obtained in $171 \AA, 195 \AA, 284 \AA$ and $304 \AA$ lines. For this analysis the data were transformed into synoptic maps for each of the spectral lines, and for the 195/171 line ratio which is an index of the coronal temperature. The synoptic maps reveal stable longitudinal structures in the coronal intensities and temperature, which are related to large-scale magnetic field structures. We discuss the relation between the coronal and photospheric magnetic structures obtained from the $\mathrm{SOHO} / \mathrm{MDI}$ data, and compare the rotation rates of these structures with the rotation profile of the solar interior in order to determine the possible origin of the coronal structures.
\end{abstract}

\section{EIT synoptic maps}

Synoptic maps of EUV images in three lines Fe and HeII $(171 \AA, 195 \AA, 284 \AA$ and $304 \AA)$ are represented by values of the line intensity centered on the central meridian $\left(I_{\|}\right)$. The original images of $1024 \times 1024$ pixels were binned to $512 \times 512$ pixels, and then each pixel in rectangular coordinates was transformed into Carrington coordinate system which is based on the sunspot rotation at the latitude of $16^{\circ}$ with synodic period of approximately 27.28 days. For synoptic purposes the images of the Sun with 4 EUV filters are taken typically 4 times a day. The synoptic maps are constructed using longitudinal stripes $16^{\circ}$ wide centered on the central meridian for each image. If an EIT image was missing, we used more wide longitudinal stripe from the previous image to cover missing values of $I_{\|}$. We have constructed the synoptic map for Carrington rotation from CR1911 to CR1959. The resolution of these maps is $1^{\circ}$ in both longitude from $0^{\circ}$ to $360^{\circ}$ and latitude from $-65^{\circ}$ to $65^{\circ}$. Examples of EIT synoptic maps for $171 \AA, 195 \AA, 284 \AA$ and $304 \AA$ for CR1935 are presented in Figure 1 . 

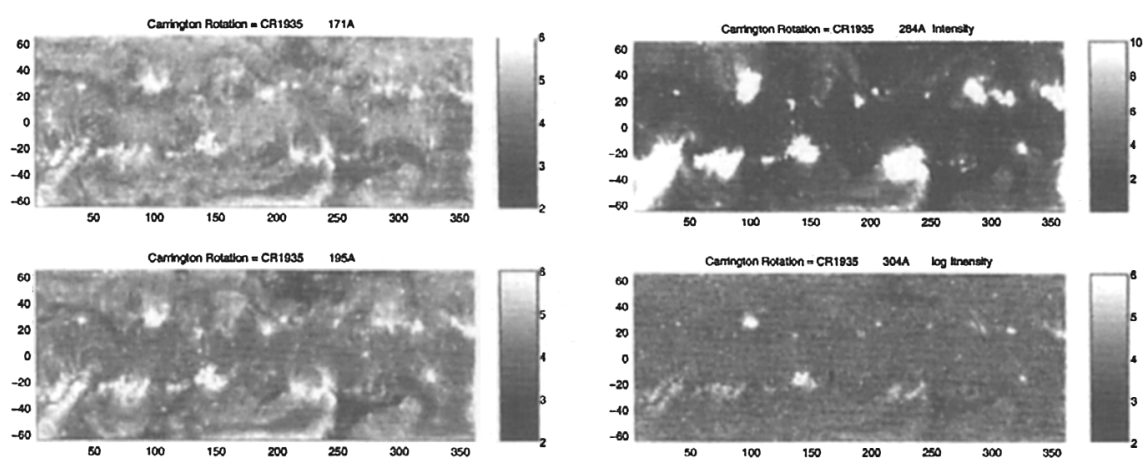

Figure 1. EIT synoptic maps for Carrington rotation 1935 in 4 lines: FeIX,X (upper left panel); Fe XII (bottom left panel); FeXV (upper right panel) and HeII (bottom right panel).

\section{Connection between magnetic field and heating of the coronal structures}

The ratio of the Fe XII $(195 \AA)$ and Fe IX,X $(171 \AA)$ fluxes is predominantly sensitive to coronal structures with the plasma temperature from 1 to $2 \mathrm{MK}$ (Moses et al, 1997). The ratio maps generally outline closed field regions. Active regions are seen as the hottest structures in the ratio images. Coronal holes are clearly defined as cold, open magnetic field regions in the ratio images.

Both magnetic and coronal $\left(I_{195} / I_{171}\right)$ synoptic maps during the transition from cycle 22 to cycle 23 and beginning of cycle 23 are shown in Figure $2 \mathrm{a}$. The coronal synoptic maps (right tall panel) clearly show the same active longitudes as the magnetic maps (Benevolenskaya et al 1999). However, the large-scale stable longitudinal patterns are better seen on the coronal maps than on the magnetic maps. During this period the magnetic structure of the Sun was determined by the interaction between the 'old' and 'new' magnetic fluxes: the 'old' flux was concentrated in two active longitudinal zones, and most of the initial 'new' flux emerged in the same zones (Benevolenskaya et al, 1999).

\section{Rotations of the coronal longitudinal structures.}

We have determined the sidereal rotation rate of $4^{\circ}$-wide latitudinal zones near the equator (the 'old' flux zone) and at $30^{\circ}$ (where the 'new' flux appeared) using the data for the current cycle. The rotation rate of magnetic flux was determined separately for the northern and southern hemispheres. Figure $2 \mathrm{~b}$ shows the rotation of the magnetic fluxes and coronal structures. It appears that the nearequatorial high-temperature coronal structures on average rotate approximately $1.7 \mathrm{nHz}$ slower than the magnetic 'old' flux of the active zone at the same latitude. In the $30^{\circ}$ zone this relation is reversed: the coronal structures rotate faster than the 'new' magnetic flux by $1.2 \mathrm{nHz}$. This result is consistent with the idea that the corona rotates more rigidly than the photosphere. However, the mechanism of this is not understood. In Figure $2 \mathrm{~b}$ we compare the rotation rates with the internal rotation determined by helioseismology. 

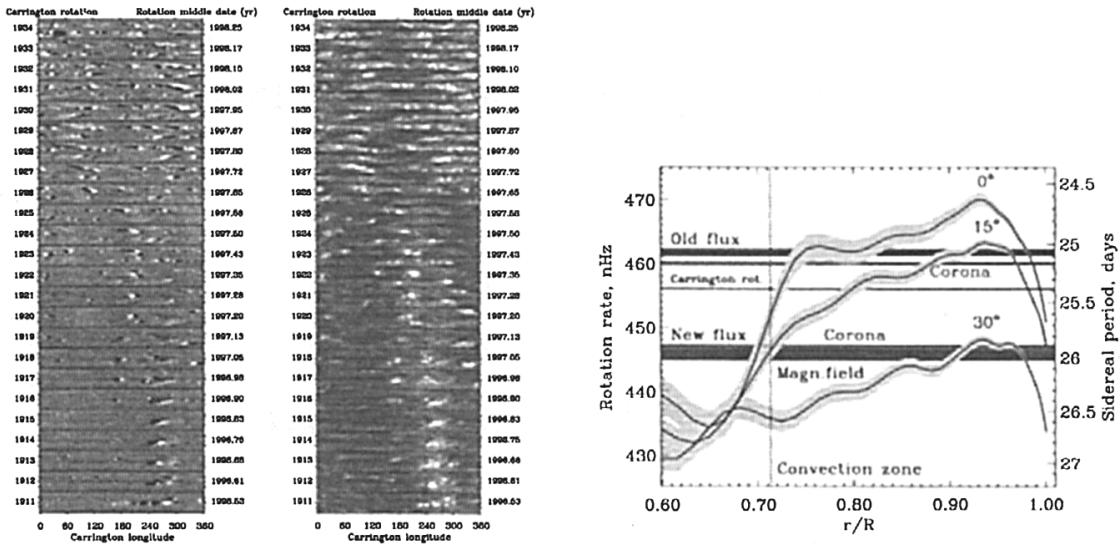

Figure 2. a) Synoptic maps of the solar magnetic field (left panel) and the EIT $195 \AA / 171 \AA$ line ratio (right panel) for Carrington Rotations 1911 to 1934 during the activity minimum between solar cycles 22 and 23 . b) Rotation rate inside the Sun determined by helioseismology as a function of radius at three latitudes, $0^{\circ}, 15^{\circ}$ and $30^{\circ}$ (solid curves with yellow areas indicating $1 \sigma$ errors). The horizontal blue areas show the rotation rates of the old magnetic flux in the latitude range $1-5^{\circ}$, and the new magnetic flux in the range $28-32^{\circ}$. The horizontal green lines shows the rotation rate of the corresponding long-living coronal temperature structures. The horizontal black line shows the Carrington rotation rate, $456.03 \mathrm{nHz}$ (sidereal period 25.38 days).

\section{Conclusions}

Studying rotation of the 'old' and 'new' fluxes and comparing their rotation rates with the internal rotation obtained by helioseismology we have suggested that both fluxes are probably generated in a narrow low-latitude zone of the tachocline (Benevolenskaya et al. 1999) - the region of the steep radial gradient of solar rotation at the bottom of the convection zone, where the magnetic flux is probably generated (e.g. Parker, 1993). Moreover, the longitudinal coronal structures heated up to $2 \mathrm{MK}$ show more rigidly rotation to compare with rotation of the pattern of the magnetic field during the rise of the cycle 23 for both 'old' and 'new' fluxes.

\section{References}

Benevolenskaya, E.E., Hoeksema, J.T., Kosovichev, A.G., and Scherrer, P.H. 1999, The interaction of the new and old magnetic fluxes at the beginning of solar cycle 23, Astrophys.J. Lett., 517, L163.

Moses, D., Clette, F., Delaboudiniere, J.-P. et al. 1997, EIT Observations of the Extreme Ultraviolet Sun, Solar Physics, 175, 571.

Parker, E. N. 1993, A solar dynamo surface wave at the interface between convection and nonuniform rotation, Astrophys. J., 408, 707. 